\title{
RAPIDLY PROGREDIATING AORTIC VALVE INFECTIVE ENDOCARDITIS IN AN INTRAVENOUS DRUG USER TREATED BY ANTIBIOTICS AND SURGERY
}

\author{
Malkia S. Swedi', Pudil Radek', Jiři Mand'ák²
}

University Hospital and Charles University in Prague, Faculty of Medicine in Hradec Králové, Czech Republic: First Department of Internal Medicine - Cardioangiology ${ }^{1}$; Department of Cardiac Surgery ${ }^{2}$

Summary: We report the case of a 22-year old male, a self-confessed recreational drug user who developed cardiogenic shock because of severe destruction of the aortic valve by rapidly progressive aortic valve endocarditis. The disease progression was acute; in a matter of days, the clinical manifestations were life-threatening necessitating urgent aortic valve replacement surgery. Cultivation revealed Streptococcus viridans as the microbial agent. Subsequent recovery with antibiotic treatment was without complication. This case report shows that immediately performed transoesophageal echocardiography and early consultation with a cardiac surgeon has fundamental importance in diagnosis and management of acute infective endocarditis in haemodynamically instable patients.

Key words: Infective endocarditis; Aortic valve; Drug user; Replacement surgery

\section{Introduction}

Infective endocarditis (IE), is a microbial infection affecting mainly the blood facing surfaces of the heart (13). The left-sided valves; both mitral and aortic are more frequently involved accounting for $92 \%$ of reported cases of IE while those on the right; tricuspid and pulmonary valves account for only $8 \%$ of reported cases (10). In intravenous drug users (IVDUs), IE presents as one of the most severe complications and the earliest cases were reported in United States in the 1950 s. In $70 \%$ of IE, involvement of the tricuspid valve was reported and in $60-70 \%$ of total infection the etiological agent was shown to be methicillin sensitive Staphylococcus aureus $(7,16)$. We report here a rare case of isolated aortic valve endocarditis in a recreational methamphetamine and marijuana drug user, whose disease rapidly progressed as a result of complete valve destruction which necessitated urgent cardiosurgery after developing cardiogenic shock.

\section{Case Report}

A 22 year-old male and a recreational drug user, presented with fever $\left(38.5^{\circ} \mathrm{C}\right)$, left calf muscle pain, chest pain and breathlessness. A week earlier he reported suffering from back pain, profuse sweating, chills, lower limbs edema, low grade fever, a painful first toe, and progressive muscle weakness which worsened with endurance activities. At admission into his district hospital, the patient suffered from dyspnea and tachycardia related to incipient heart failure, and within a few hours he developed cardiogenic shock. This progressed to cardiac arrest which necessitated resuscitation and administration of catecholamines and he was later transferred to our cardiology intensive care unit. At admission, he was unconscious, artificially ventilated, with blood pressure of 211/40 $\mathrm{mmHg}$, and pulse $160 \mathrm{bpm}$. On arrival, biochemical analysis showed serum electrolytes to be within normal ranges, creatinine $134 \mu \mathrm{mol} / \mathrm{L}$, CRP $153.3 \mathrm{mg} / \mathrm{L}$, TnT 0.682, WBC $33.25 \times 10^{9} / \mathrm{L}, \mathrm{RBC}$ $4.25 \times 10^{12} / \mathrm{L}, \mathrm{Hb} 123 \mathrm{~g} / \mathrm{L}$, Platelets $440 \times 10^{9} / \mathrm{L}$, blood pH 7.144, HIV negative, HAV, HBsAg and HCV negative. Blood culture samples were taken at the time of admission, and Streptococcus viridans was found. Chest X-ray showed bilateral infiltrates with little pleural effusion. An immediate transoesophageal echocardiography was performed which revealed a hemodynamically significant aortic valve insufficiency (Fig. 1B) and vegetations (Fig. 1A) on a severely destructed aortic valve (Fig. 1C, 1D). Urgent mechanical valve replacement cardiosurgery was indicated as the optimal treatment option, and was carried out within 90 minutes of his arrival. After the operation the patient suffered from pericardial hemorrhagic effusion, which was drained after 5 days. The patient was further treated with a combination of cefotaxim and gentamicin for two weeks in the intensive care unit and his state progressively improved with signs of inflammation subsiding. He was then transferred to the general ward and treatment was continued, with antibiotics, rehabilitation and anticoagulation, and he was discharged after 7 weeks with negative blood cultures, normal C-reactive protein level, no signs of endocarditis on echocardiography, no signs of heart failure and normalisation of CRP. 


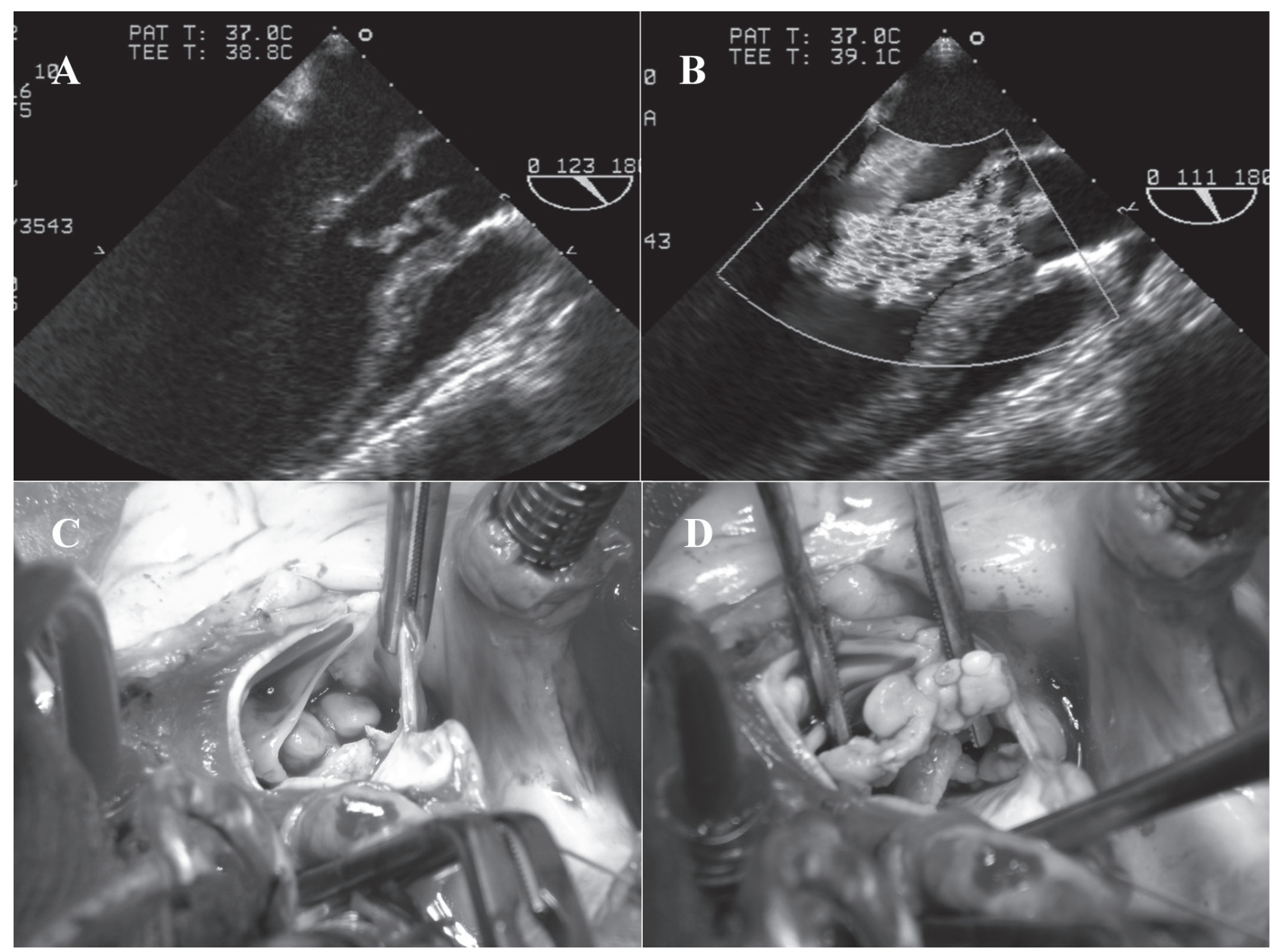

Fig. 1: A) Severe aortic valve regurgitation transoesophageal view, color flow Doppler; B) Aortic valve with vegetation, transoesophageal view; C) Aortic valve with destruction of cusps, peri-operative photo; D) Aortic valve with large vegetation, peri-operative photo

\section{Discussion}

Infective endocarditis is a potentially life-threatening condition and leads to increased morbidity and mortality. The incidence rate in the general population is between 1.7 to 6.2 cases per 100,000 (9) and the number affected has been relatively stable for the past three decades $(12,17)$. In spite of this, the demographic make-up of the population at risk has changed, with IE increasingly affecting elderly patients undergoing hospital procedures (12). In addition, increased numbers of IE infections have been shown in intravenous drug users $(2,12)$, in nosocomial settings $(8$, $12)$, and in chronically hemodialysed patients $(1,12)$.

Dressler and Roberts showed, that in eighty IVDUs, the first episode of IE involved a single right-sided cardiac valve in 24 patients $(30 \%)$; both a right- and a left-sided valve in 13 patients (16\%); a single left-sided valve in 33 patients (41\%); and both left-sided valves in 10 patients (13\%) (11). Merle et al showed, that right-sided valvular involvement has the highest incidence with the tricuspid valve involved in $60-70 \%$ of cases and involvement of the pulmonary valve in only $2 \%$ of cases (16). In one study of IVDUs, vegetations were found in the tricuspid valve in 127 cases, in the pulmonary valve in 4 cases and vegetations were found on both valves in only 1 case (5). In a review study of right sided IE, 85 out of 86 patients were found to involve solely the tricuspid valve and only 1 with the pulmonary valve (2). Additionally, aortic and mitral valve involves $30-40 \%$ of IE cases. Furthermore, more than one valve may be infected concurrently in either side of the heart (16).

The pathogenesis which tends to drive involvement of right-sided IE in IVDUs is not yet fully elucidated. Most likely, the damage to right-sided valves follows from the bacterial load of injected particulate matter and secondly, it may be due to compromised host immune defense mechanisms $(6,12)$.

Currently, infection with Staphylococcus aureus and Enterococcus spp. account for more than $80 \%$ of etiological 
agents causing IE $(11,16)$. The rest of infections are mainly due to Streptococci and Pseudomonas species with lowest numbers being accounted for by Candida and Asperillus spp. $(11,16)$.

The definitive diagnosis of endocarditis is based on Duke's criteria (12). However, more recently, echocardiography appears to be playing an increasingly important role in early diagnosis of IE. This has the advantage of allowing prompt and specific treatment, although it is associated with the drawback in that it can demonstrate valvular lesions of only $2-3 \mathrm{~mm}$ or more in size $(7,12)$. It can also serve as a confirmatory tool to detect endocardial involvement even in the absence of positive blood cultures (7). In the acute phase, aortic valve infective endocarditis may present with vegetations of various sizes which consist of septic thrombi entrapping microorganisms and neutrophilic infiltrates. In such cases, echocardiography is unrivalled in being able to detect early vegetative lesions, destructed and ulcerated leaflets associated with valve regurgitation, prosthetic valve dehiscence, as well as abscess formation (12). In addition, by utilising a molecular biology approach in the use of the polymerase chain reaction on samples of either embolic material or excised valve, early microbiological diagnosis of infective valve endocarditis can be established $(4,12)$.

Pulmonary artery embolism and infarcts, pneumonia, and lung abscesses are complications resulting from rightsided endocarditis $(3,12)$. Left-sided endocarditis can be complicated by systemic embolism and by cerebral, myocardial, splenic, kidney, and intestinal infarcts or abscesses leading to organ failure (12). The most prevalent extra-cardiac complications associated with IE are embolic events with an incidence ranging from $22 \%$ to $43 \%$ (3). Extensive and profuse valvular vegetations from Streptococcus viridans infection seem to be a serious risk factor associated with embolism (3). Complications associated with the aortic root carry an increased frequency of operative mortality and postoperative regurgitation (15). Furthermore, there have been a few reports of extension of aortic valve infective endocarditis to the mitral valve. This lesion progression occurs through mitro-aortic fibrous continuity, leading to the development of a complication manifested as anterior leaflet septic aneurysm of the mitral valve (a socalled kissing lesion), with or without perforation.

According to the guidelines of ESC and guidelines of Czech Society of Cardiology, antibiotic therapy remains the central axiom of IE treatment; high dose intravenous penicillin or ceftriaxone or cefotaxim in combination with aminoglycosedes is the fundamental therapy for penicillinsusceptible streptococcal endocarditis (12). A combination regimen of amoxicillin or ampicillin with gentamicin or netilmicin is appropriate for enterococci (12). In cases of patients with allergy to penicillin, vancomycin is substituted $(12,14)$. In methicillin-susceptible staphylococcal native-valve infection, flucloxacillin, oxacillin or vancomycin combined with gentamicin is recommended (12).
Antibiotic therapy is prolonged, generally for two to six weeks (12). Proven fungal endocarditis requires specific treatment with amphotericin B (12). Surgical debridement of infected material and artificial heart valve replacement with either mechanical or bioprosthetic valves plays a major role in patients who are unresponsive to antibiotic therapy, in those developing cardiac failure resulting from valvular destruction, and in cases presenting with complications (12).

Surgical therapy during the active phase of the disease is associated with significant risk. Operative mortality in IE lies between 5 and $15 \%$. When surgery must be performed within the first week of antimicrobial therapy, a recent study showed that in-hospital mortality is $15 \%$, with risks of recurrence and non-infective post-operative valvular dysfunction of 12 and 7\%, respectively. Replacement of the aortic valve using a mechanical or biological prosthesis is the technique of choice (12).

Surgery is justified in patients with high-risk features which make the possibility of cure with antibiotic treatment unlikely and who do not have co-morbid conditions or complications which make the prospect of recovery remote. According to current European Society of Cardiology guidelines, surgery needs to be performed on an emergency (within $24 \mathrm{~h}$ ) basis in patients with: [1] aortic or mitral IE with severe acute regurgitation or valve obstruction causing refractory pulmonary oedema or cardiogenic shock, [2] aortic or mitral IE with fistula into a cardiac chamber or pericardium causing refractory pulmonary oedema or cardiogenic shock (12).

Our case report showed that in haemodynamically unstable patients with infective endocarditis: [1] the immediately performed transoesophageal echocardiography has fundamental importance in diagnosis and management, [2] early consultation with a cardiac surgeon is highly recommended in order to determine the best therapeutic approach.

\section{Conclusion}

Although major advances have taken place in both medical and surgical interventions, IE still remains a serious disease associated with high incidences of morbidity and mortality. From a clinical viewpoint, it is of utmost importance to consider IE in every patient presenting with clinical features of fever, septicemia, and new regurgitant heart murmurs. Early echocardiography may save time in identifying smaller vegetations even in the absence of proven microbiological cultures. Early aggressive antibiotic therapy and prompt surgical interventions can save lives.

\section{Acknowledgements}

This case report was supported by the Research Project PRVOUK P37/03. Catherine Mc Grath M.D., Ph.D. is acknowledged for linguistic editing of the manuscript. 


\section{References}

1. Abbott KC, Agodoa LY. Hospitalizations for bacterial endocarditis after initiation of chronic dialysis in the United States. Nephron 2002; 91: 203-9.

2. Addai T, Anand A, Freels S, Mathew J, Maheshwari P, Morrobel A. Clinical features, site of involvement, bacteriologic findings, and outcome of infective endocarditis in intravenous drug users. Arch Intern Med 1995; 155: 1641-8

3. Ballard D, Murphy JG, Stekelberg JM, et al. Emboli in infective endocarditis: the prognostic value of echocardiography. Ann Intern Med 1991; 114: 635-40.

4. Becker K, Breitkopf C, Hammel D, Peters G, Scheld HH. Impact of a molecular approach to improve the microbiological diagnosis of infective heart valve endocarditis. Circulation 2005; 111: 1415-21.

5. Berger M, Hectit S. Right sided endocarditis in intravenous drug users, prognostic features in 102 episodes. Ann Internal Medicine 1992; 117: 560-6.

6. Brd Munt, Ross Moss.; Injection drug use and right sided endocarditis; Heart 2003; 89: 577-81

7. Bright DK, Durack DT, Lukes AS. New criteria for diagnosis of infective endocarditis: utilization of specific echocardiographic findings. Duke Endocarditis Service. Am J Med 1994; 96: 200-9.

8. Burkert T, Watanakunakorn C. Infective endocarditis at a large community teaching hospital, 1980-1990. A review of 210 episodes. Medicine (Baltimore) 1993; 72: $90-102$

9. Calderwood SB, Mylonakis E. Infective endocarditis in adults. N Engl J Med 2001; 345: 1318-330.
10. Chetchotisakd P, Klugboonkrong V, Loapibon M, Pachirat O, Tantisivin C, Tawelsangsuksakul P. Infective endocarditis: prevalence, characteristics and mortality in khon kaen 1990-1999. J Med Assoc Thai 2002; 85 (1): 1-10.

11. Dressler FA, Roberts WC. Infective endocarditis in opiate addicts: analysis of 80 cases studied at necropsy. Am J Cardiol 1989; 63(17): 1240-57.

12. Habib G, Hoen B, Tornos P et al. Guidelines on the preven tion, diagnosis, and treatment of infective endokarditis (new version 2009). European Heart Journal 2009; 30: 2369-2413.

13. Follath F, Gutschik E, Horstkotte D, et al. Guidelines on prevention, diagnosis and treatment of infective endocarditis executive summary. The task force on infective endocarditis of the European Society of Cardiology. Eur Heart J 2004; 25: $267-76$

14. Infective endocarditis: Diagnosis, antimicrobial therapy and management of complications: A statement from the committee on rheumatic fever, endocarditis and Kawasaki disease. Circulation 111: 2005; e394-e434.

15. John RMJ, Sturridge MF, Swanton RH, Treasure T. Aortic root complications of infective endocarditis: influence on surgical outcome. Eur Heart J 1991; 12: 241-8.

16. Merle A, Mrinkak JA. Infective endocarditis. In: Valentin FR, Wayne A, et al eds Hurst's The Heart. 10th edition. Mc Graw - Hill 2001. Vol. 2; 2087-125.

17. Moreillon P, Que YA. Infective endocarditis. Lancet 2004; 363: 139-49.

Received: 10/05/2010

Accepted in revised form: 09/05/2012

\section{Corresponding author:}

Prof. Radek Pudil, M.D., Ph.D., University Hospital in Hradec Kralove, 1st Department of Internal Medicine, Charles University in Prague, Faculty of Medicine in Hradec Králové, Sokolská 581, 50002 Hradec Králové, Czech Republic; e-mail: pudilradek@yahoo.com 